DOI: https://doi.org/10.11144/Javeriana.umed59-1.taba

\title{
Terapia de remplazo nicotínico asociada con reducción gradual del consumo de cigarrillos como estrategia de cesación de tabaco. Reporte de caso
}

Nicotinic Replacement Therapy Associated with Gradual Reduction as a Strategy of Smoking Cessation. Case report

\author{
Nelci Becerra a \\ Clínica para Dejar de Fumar, Javesalud . \\ Pontificia Universidad Javeriana, \\ Colombia \\ Pilar García-Padilla \\ Clínica para Dejar de Fumar, Javesalud - \\ Pontificia Universidad Javeriana, \\ Colombia

\section{RESUMEN} \\ El habitual proyecto de definir una fecha para dejar de fumar \\ abruptamente ha sido refutado por muchos pacientes que han mostrado \\ interés por reducir su consumo de modo gradual. La reducción progresiva \\ utilizando terapia sustitutiva con nicotina previa a la cesación permitiría \\ tratar a un mayor número de fumadores. El artículo presenta el caso de un \\ paciente con alta dependencia a la nicotina, interesado en dejar de fumar, \\ pero no de manera abrupta. \\ Palabras clave \\ hábito de fumar; cese del tabaquismo; reducción del daño.
}

\begin{abstract}
The usual approach to the treatment of smoking to set a date to stop smoking abruptly has been refuted by many patients who show interest in reducing their consumption gradually. The progressive reduction using nicotine replacement therapy prior to cessation would treat a greater number of smokers. It's the case of a patient with high nicotine dependence, interested in quitting but not abruptly.

Keywords

smoking; smoking cessation; harm reduction.
\end{abstract}

\section{Introducción}

El tabaquismo es la primera causa de enfermedad y muerte prevenible en el mundo $(1,2,3)$. Además de las consecuencias negativas para la salud en las personas fumadoras y expuestas

a Autor de correspondencia. Correo electrónico: becerra.n@javeriana.edu.co

Cómo citar: Becerra N, García-Padilla P. Terapia de remplazo nicotínico asociada con reducción gradual del consumo de cigarrillos como estrategia de cesación de tabaco. Reporte de caso. Univ Med. 2018;59(1):1-6. doi: https://doi.org/10.11144/ Javeriana.umed59-1.taba a humo de segunda mano, las consecuencias para el medio ambiente son también tema de preocupación (4). En Colombia, según el Estudio Nacional de Salud Mental 2015, la prevalencia de consumo de tabaco alguna vez en la vida en adolescentes es del 5,8\%, y de este porcentaje más de la mitad de los adolescentes continúa fumando (56,7\%). De los adultos jóvenes (18 a 44 años), el 15,9 \% ha fumado cigarrillos 
alguna vez en su vida y el $67,8 \%$ continúa su consumo. Se estima que en Colombia anualmente mueren alrededor de 21.000 personas por enfermedades asociadas al consumo de cigarrillo (5), sin que hasta el momento exista disponibilidad amplia de medidas clínicas para su control en el Sistema General de Seguridad Social en Salud.

Teniendo en cuenta el carácter adictivo de los productos del tabaco, en su mayoría los fumadores necesitan apoyo para dejar de fumar, y aunque no son comparables, las encuestas de población indican que, cada año, aproximadamente un tercio de los fumadores trata de dejar de fumar y que en la mayor parte de los casos lo hace sin ayuda. Sin embargo, solo un pequeño porcentaje de fumadores (1-3 $\%)$ logra una abstinencia duradera (por lo menos de 12 meses) aplicando únicamente la fuerza de voluntad (6).

Ante esta situación, el tratamiento del tabaquismo es recomendado por la Organización Mundial de la Salud como parte integral y esencial de los servicios de prevención y control de las enfermedades no transmisibles en la atención primaria en salud (7). Los programas de cesación tabáquica abarcan estrategias de intervención terapéutica multicomponente (8): la consejería breve e intensiva y la terapia farmacológica - terapia de remplazo nicotínico (TRN), bupropión, nortriptilina y vareniclina-, que son eficaces en la cesación de tabaquismo (9). Así mismo, la intervención psicológica de tipo cognitivo-comportamental ha mostrado ser eficaz para dejar de fumar $(10,11,12)$.

Una de las estrategias de cesación de tabaco ampliamente utilizada en el contexto clínico es la de proponerle al paciente que escoja una fecha para dejar de fumar. Desde el punto de vista individual, es posible que, en determinadas circunstancias, la abstinencia total no sea un objetivo realista y se pueda plantear una estrategia de reducción del daño. Una encuesta realizada en España mostró que del total de fumadores de la población general, solo el 26,6\% quería dejar de fumar; de ellos el 52 $\%$ era partidario de intentarlo abruptamente, en tanto que el $48 \%$ restante indicó que le gustaría reducir su consumo de cigarrillos como paso previo a la cesación. Por otro lado, dentro del grupo de fumadores que no quería dejar de fumar, el $84 \%$ no mostró intención de cambiar su actitud; mientras que el $16 \%$ indicó como alternativa la reducción del número de cigarrillos (13). A la vista de estos datos, se ha recomendado buscar estrategias terapéuticas de cesación tabáquica que se adapten a las necesidades y motivaciones de los pacientes fumadores $(14,15,16)$. El objetivo de este artículo es presentar un caso clínico y los resultados de la intervención terapéutica en un paciente fumador utilizando la estrategia de reducción progresiva de consumo asociado con el uso de TRN como paso previo a la cesación tabáquica.

\section{Caso clínico}

El caso corresponde a un hombre de 55 años de edad con un nivel educativo de posgrado que consultó el Programa de Cesación de Tabaco ofrecido en un servicio médico universitario (consentimiento informado firmado por el paciente). Refirió estar muy interesado en dejar de fumar en los siguientes treinta días si pudiera hacerlo fácilmente, aunque no estaba seguro de lograrlo.

Respecto a la conducta de fumar, contó que inició a los 13 años. Fuma el primer cigarrillo en cuanto se despierta y consume en promedio treinta cigarrillos al día. Había realizado seis intentos para dejar de fumar, con un máximo de seis meses de abstinencia. Había utilizado como estrategias de cesación la fuerza de voluntad y medicamentos como los chicles de nicotina y bupropión; sin embargo, había presentado recaídas asociadas con altos niveles de estrés y crisis vital por la muerte de su padre. En esta ocasión, sus principales motivaciones para dejar de fumar son garantizar mejores condiciones de salud y tener una mayor percepción de control respecto del hábito.

En cuanto a sus antecedentes médicos, refirió un accidente isquémico transitorio tres años atrás. Negó el consumo de sustancias psicoactivas. En el examen físico de ingreso, el paciente se encontraba en buen estado general. 
Tensión arterial: 120/80. Frecuencia cardiaca: 78 $\mathrm{lpm}$. Frecuencia respiratoria: $16 \mathrm{rpm}$. Oximetría de pulso: $94 \%$. Índice de masa corporal: 22,5 $\mathrm{kg} / \mathrm{m}^{2}$. El examen físico estaba dentro de los límites normales. La evaluación interdisciplinaria permitió identificar tabaquismo muy grave, alta dependencia física a la nicotina y dependencia psicológica severa. En relación con su motivación para el cambio, el paciente se encontraba en la etapa de preparación según el modelo transteórico de Prochaska y presentaba alta motivación para dejar de fumar.

En la evaluación psicosocial se identificó un alto nivel de estrés percibido, asociado con un bajo nivel de autoeficacia, y una disfunción familiar moderada. La cooximetría reportó un nivel de 21 ppm de monóxido de carbono en el aire espirado, cifra concordante con los hallazgos encontrados en el test de Fagerström (alta dependencia física a la nicotina). Los resultados encontrados en la evaluación clínica interdisciplinaria se pueden ver en la tabla 1 .

Tabla 1. Evaluación del estatus tabáquico del paciente

\begin{tabular}{|l|l|l|}
\hline Datos clínicos & Puntaje & Interpretación \\
\hline Años de fumador & 42 & \multirow{2}{*}{ Tabaquismo muy grave } \\
\hline Número de cigarrillos al dia & 30 & \\
\hline Índice paquetes/año & 63 & Alta dependencia física a la nicotina \\
\hline Test de Fagerström & 9 & Dependencia psicológica severa \\
\hline Test Glover-Nilsson & 32 & Motivación alta para dejar de fumar \\
\hline Test de Richmond & 7 & Preparación \\
\hline Etapa de cambio (Prochaska) & $\begin{array}{l}\text { En el próximo } \\
\text { mes }\end{array}$ & Disfunción familiar moderada \\
\hline APGAR familiar & 13 & Riesgo de depresión \\
\hline TG para depresión & 3 & Riesgo de ansiedad \\
\hline TG para ansiedad & 4 & Sin depresión \\
\hline Test de Beck (depresión) & 8 & Baja ansiedad (no problemático) \\
\hline Test STAI (ansiedad) & E9-R12 & Alto nivel de estrés percibido \\
\hline Escala de Estrés Percibido & 42 & Bajo nivel de autoeficacia \\
\hline Escala de Autoeficacia & 12 & $\begin{array}{l}\text { Fumador frecuente. Niveles 4 veces } \\
\text { superiores a lo normal. Importante } \\
\text { adicción a la nicotina }\end{array}$ \\
\hline Cooximetría & 21 ppm \\
\hline
\end{tabular}

Como parte de la evaluación médica, se solicitó una espirometría para tamización de enfermedad pulmonar obstructiva crónica, que obtuvo como resultado: leve alteración ventilatoria de tipo obstructivo, que responde significativamente con la administración de B2 inhalado; por tal motivo, se solicitó una valoración interdisciplinaria por parte del servicio de neumología. Adicionalmente, se recomendó vacunación contra influenza y neumococo, así como valoración por parte del servicio de odontología.

\section{Intervención terapéutica}

Después de la evaluación clínica del paciente y considerando tanto sus necesidades como sus preferencias, se inició un plan de reducción gradual del número de cigarrillos diarios utilizando la TNR como paso previo a la cesación tabáquica, teniendo en cuenta el registro diario de consumo de cigarrillos. Así, se inició un esquema de reducción gradual de cigarrillos: un cigarrillo menos cada dos días, asociado con TRN. Por antecedente de hipopersistente con los chicles de nicotina, se seleccionaron los parches transdérmicos de nicotina. Se inició con una dosis de $5 \mathrm{mg} / 16 \mathrm{~h}$ y uso diario durante 4 semanas (el parche se retira en la noche).

Al mes de iniciar la intervención terapéutica, el paciente alcanzó una disminución del 50 \% del consumo inicial. Para el segundo mes se aumentó la dosis del parche de nicotina a $10 \mathrm{mg} / 16 \mathrm{~h}$. Este esquema continuó a la par con la disminución gradual del consumo de cigarrillos. Al iniciar el tercer mes de tratamiento, el paciente solamente estaba fumando 2 cigarrillos diarios, y al sentirse preparado para dejar de fumar, logró dejarlo y se continuó con el uso de parches de nicotina en la misma dosis de $10 \mathrm{mg} / 16 \mathrm{~h}$ durante 4 semanas más. Para iniciar el cuarto mes de tratamiento, se redujo la dosis del parche de nicotina a $5 \mathrm{mg} / 16$ $\mathrm{h}$ durante 4 semanas, y al iniciar el quinto mes de intervención se suspendió la TRN.

La intervención psicológica consideró tres momentos del proceso: preparación, abandono y mantenimiento. En cada uno de ellos se utilizaron herramientas específicas. Así, se trabajó con entrevista motivacional, para favorecer el inicio y persistencia en la cesación, y con autobservación (registros), para promover la toma de conciencia respecto de la magnitud del consumo. En relación con el abandono y mantenimiento, se incluyeron estrategias de control de estímulos, autocontrol y entrenamiento en solución de problemas, así 
como elementos específicos para favorecer la prevención de recaída.

\section{Evolución clínica}

Durante el primer mes se realizaron sesiones de seguimiento semanal, evaluando signos vitales, peso, presencia de síntomas y signos de abstinencia a la nicotina, deseo de fumar, apropiación de recursos psicológicos, adherencia al tratamiento y posibilidad de efectos secundarios a la terapia farmacológica. Se utilizó la Escala Minnesota de Abstinencia a la Nicotina para evaluar síndrome de abstinencia, y la Escala Single Rating of Craving, para evaluar el deseo de fumar. El paciente presentó durante las primeras dos semanas dificultad para dormir, síntoma que fue disminuyendo progresivamente, posterior a recomendaciones sobre higiene del sueño. El nivel de cooximetría también disminuyó de manera concordante con la reducción del número de cigarrillos (reporte de cooximetría al mes de inicio de la intervención terapéutica de $10 \mathrm{ppm})$.

Durante los meses segundo y tercero se realizaron controles quincenales, sin encontrar signos de síndrome de abstinencia. Posteriormente, se programaron seguimientos con una periodicidad mensual. El paciente presentó una evolución satisfactoria, con adecuada adherencia al tratamiento implementado. En el control a los seis meses de haber dejado de fumar, el paciente refirió sentirse muy satisfecho del proceso alcanzado y la cooximetría de control fue de $3 \mathrm{ppm}$, siendo un marcador real de abstinencia.

\section{Discusión}

Una posible barrera para algunos fumadores en su objetivo de alcanzar la cesación del cigarrillo es el habitual planteamiento de abandono abrupto, al definir una fecha para dejar de fumar. Pocos fumadores están dispuestos a dejar de fumar en una fecha específica, mientras que un mayor número de fumadores se muestra interesado en la reducción progresiva (17). La reducción del consumo de tabaco podría representar un primer paso hacia la cesación definitiva, habida cuenta que el fumador puede experimentar un grado de éxito al controlar su comportamiento, lo cual puede motivarlo para alcanzar su objetivo terapéutico (18).

Durante los últimos años se han realizado un gran número de ensayos clínicos en los que se ha analizado la eficacia de la reducción como paso previo a la cesación $(19,20,21,22)$, y se ha encontrado que reducir el número de cigarrillos consumidos al día no solo incrementa la motivación para realizar intentos de abandono definitivo del tabaco, sino que también aumenta las posibilidades de conseguirlo (OR: 2,96; IC 95 \%: 1,90-4,61) $(23,24,25)$. Dos metanálisis han confirmado estos hallazgos: el primero realizado sobre un total de 8 ensayos clínicos halló que aquellos fumadores que reducían el consumo del tabaco utilizando TRN multiplicaban por 1,9 sus posibilidades de abandonarlo definitivamente en comparación con los que utilizaban placebo (OR: 1,90; IC $95 \%$ : 1,5-2,5) (26); el segundo, realizado sobre un total de cinco ensayos clínicos, reportó cifras superiores (OR; 2,5; IC $95 \%$ : 1,7-3,7) (27). Otro aspecto importante para tener en cuenta es la seguridad del uso de TRN concomitantemente con cigarrillos; al respecto, los estudios concluyen que es seguro, pues los efectos adversos que aparecen son similares a los que se manifiestan en los estudios en los que la TRN se utiliza sin consumo de tabaco y, además, en ningún caso se observaron signos o síntomas de intoxicación nicotínica $(25,28,29)$.

Los resultados descritos en este caso permiten señalar que la combinación de TRN asociado con la disminución progresiva de consumo de cigarrillos, incluyendo el componente de intervención psicológica, favorece la cesación tabáquica en pacientes con intentos fallidos previos, lo que puede estar asociado con el incremento en la percepción de autoeficacia y control por parte del paciente. Por otra parte, es pertinente plantear la indicación de esta intervención terapéutica que combina elementos tanto farmacológicos como no farmacológicos en dos grupos de pacientes: fumadores que no quieren dejar de fumar pero que se muestran 
interesados en reducir significativamente el número de cigarrillos al día y fumadores que, queriendo dejar de fumar, encuentran muchas dificultades en conseguirlo y se muestran reticentes ante la idea de abandonar de forma brusca el consumo.

Con respecto al primer grupo, cabe anotar que este proceso terapéutico podría favorecer el cambio de intención desde la reducción hacia la cesación, pues si bien la disminución del consumo puede contribuir es necesario precisar que, independientemente del tipo de intervención seleccionado, la única manera para decrecer los riesgos ocasionados por el tabaquismo es el abandono definitivo del hábito de fumar (30).

\section{Referencias}

1. US Department of Health and Human Services. The health consequences of smoking: A report of the surgeon general. Atlanta, (GA): U.S. Department of Health and Human Services, Centers for Diseases Control and Prevention, National Center for Chronic Disease Prevention and Health Promotion, Office on Smoking and Health; 2004.

2. World Health Organization. Report on the global tobacco epidemic. Switzerland: WHO; 2011.

3. U.S. Department of Health and Human Services. The health consequences of smoking 50 years of progress: A report of the surgeon general. Atlanta, GA: U.S. Department of Health and Human Services, Centers for Disease Control and Prevention, National Center for Chronic Disease Prevention and Health Promotion, Office on Smoking and Health; 2014. Printed with corrections, January 2014.

4. American Cancer Society and World Lung Foundation. The Tobacco Atlas. 2015.

5. Pichon A, Bardach A, Caporale J, Augustovski F, Alcaraz A, Navia M, et al. Health and economic burden of tobacco use in seven Latin American countries: Results from a microsimulation Health Economic Model
(HEM). Value in Health 16. 2013:A665A728.

6. Fiore MC, Jaén CR, Baker TB, Bailey WC. Treating tobacco use and dependence: 2008 update. Clinical practice guideline. Rockville, MD: U.S. Department of Health and Human Services, Public Health Service; 2008.

7. Organización Mundial de la Salud. Strengthening health systems for treating tobacco dependence in primary care. Part 1: Training forpolicy. Washington: OMS; 2013.

8. Aveyard P, Raw M. Improving smoking cessation approaches at the individual level. Tob Control [internet]. 2012;21(2):252-57. Disponible en: http://tobaccocontrol.bmj.com/content /21/2/252.full

9. Alba LH, Murillo R, Becerra N, Páez N, Cañas A, Mosquera Ca, Castillo JS, Camacho N, Gómez J, García-Herreros P, Bernal LG. Recomendaciones para la cesación de la adicción al tabaco en Colombia. Biomédica. 2013;33:186-204.

10. Guichenez P, Clauzel I, Cungi C, et al. The contribution of cognitive-behavioural therapies to smoking cessation [in French]. Rev Mal Respir. 2007;24(2):171-82.

11. Fernández Arias I. Eficacia de la terapia cognitivo conductual, los parches de nicotina y su combinación para dejar de fumar. Tesis Doctoral, Universidad Complutense de Madrid, España; 2012.

12. Becoña E, Fernández del Río E, López A, Martínez U, Martínez C, Rodríguez RA. El tratamiento psicológico de la dependencia del tabaco: Eficacia, barreras y retos para el futuro. Papeles del Psicólogo. 2014;35(3):161-8.

13. Encuesta Millward Brown. Mayo 2006. Barcelona.

14. Fagerström KO. Interventions for treatmentresistant smokers. Nicotine Tob Res. 1999;1:S201-5.

15. Fagerström KO. Can reduced smoking be a way for smokers not interested in quitting to actually quit? Respiration. 2005;72:216-20. 
16. Ebbert JO, Hughes JR, West RJ, et al. Effect of varenicline on smoking cessation through smoking reduction: a randomised clinical trial. JAMA. 2015;313:687-94.

17. Jiménez Ruiz CA, Fagerström KO. Reducción hasta dejarlo: árbol de decisión. Prev Tab. 2006;8(Supl. 1):34-7.

18. Hughes JR, Carpenter MJ. The feasibility of smoking reduction: an update. Addiction. 2005;100:1074-89.

19. Wennike P, Danielsson T, Landfeldt B, Westin A, Tonnesen P. Smoking reduction promotes smoking cessation: results from a double blind, randomized, placebocontrolled trial of nicotine gum with 2-year follow-up. Addiction. 2003;98:1395-1402.

20. Batra A, Klingler K, Landfeldt B, Friederich HM, Westin A, Danielsson T. Smoking reduction treatment with 4-mg nicotine gum: A double-blind, randomized, placebocontrolled study. Clin Pharmacol Ther. 2005;78:689-96.

21. Rennard SI, Glover E, Leischow S, Daughton DM, Glover P, Muramoto M, Franzon M, Danielsson T, Landfeldt B, Westin A. Efficacy of nicotine inhaler in smoking reduction: a double-blind, randomized trial. Nicotine Tob Res. 2006;8:555-64.

22. Kralikova E, Kozak J, Rasmussen T, et al. Smoking cessation or reduction with nicotine replacement therapy: A placebocontrolled double blind trial with nicotine gum and inhaler. BMC Public Health. 2009;9:433.

23. Wennike $P$, Danielsson $T$, Landfeldt $B$, Westin A, Tønnesen P. Smoking reduction promotes smoking cessation: results from a double blind, randomised, placebocontrolled trial of nicotine gum with 2-year follow-up. Addiction. 2003;98:1395-1402.

24. Glasgow RE, Gaglio B, France EK, Marcus $\mathrm{Al}$, Riley KM. et al. Do behavioural smoking reduction approaches reach more or different smokers?: Two studies similar answers. Add Beh. 2006;31:510-8.

25. Hughes JR, Callas PW, Peters EN. Interest in gradual reduction. Nic Tob Res. 2007;6:671-5.
26. Stead LF, Lancaster T. Interventions to reduce harm from continued tobacco use. Cochrane Database Systems Review. 2007;18(3):CD 005231.

27. Sophia SC, Chan D. Leung A, Abdullah V, Wong A, Hedley L. A randomized controlled trial of a smoking reduction plus nicotine replacement therapy intervention for smokers not willing to quit smoking. Addiction. 106;1155-63.add

28. Jiménez Ruiz CA, Riesco Miranda JA, Ramos Pinedo A, Barrueco Ferrero M, Solano Reina S, de Granda Orive JI, et al. Recomendaciones para el tratamiento farmacológico del tabaquismo: propuesta de financiación. Arch Bronconeumol. 2008;44(4):213-9.

29. Fagerström KO, Hughes RJ. Nicotine concentrations with concurrent use of cigarettes and nicotine replacement: A review. Nic Tob Res. 2002;4:S155-61.

30. Lindson-Hawley N, Hartmann-Boyce J, Fanshawe T, Begh R, Farley A, Lancaster T. Interventions to reduce harm from continued tobacco use. Cochrane Database. Octubre, 2016. 